Ann. Zootech., 1986, 35 (4), 363-372

\title{
Suppression de la chute estivale de la production du poil chez la lapine angora par la pose d'implants de mélatonine
}

\author{
J. ROUGEOT, R.G. THEBAULT et D. ALLAIN * \\ INRA, Laboratoire des Pelages, Toisons et Fourrures, \\ 78350 Jouy-en-Josas, France \\ * Adresse actuelle: INRA, Unité de Neurobiologie de la reproduction, 78350 Jouy-en-Josas
}

\begin{abstract}
Résumé
Des lapines angora adultes élevées dans un bâtiment clos dont l'éclairage artificiel était réglé sur le photopériodisme naturel ont été traitées par des implants de mélatonine au mois de mai, 1 semaine avant la récolte de poils de printemps et pendant toute la période de croissance du pelage d'été (98 jours). Il en résulte une augmentation de $31,2 \mathrm{p} .100$ de la production de poils ainsi qu'une amélioration de 17,1 p. 100 de la compacité du pelage et de 8,3 p. 100 de la longueur des duvets, alors que les longueurs des jarres et des barbes ainsi que les diamètres des 3 catégories de poils ne sont pas modifiés. Ainsi, la pose d'implants de mélatonine au moment de la récolte de printemps permet-elle de supprimer la chute estivale de la production de poils et d'obtenir en été une récolte de poils équivalente en quantité à celles d'automne et d'hiver.
\end{abstract}

Mots clés : Lapine angora, production de poils, saison, mélatonine, structure du pelage.

\section{Introduction}

Comme la plupart des pelages des mammifères vivant en climats froids ou tempérés, celui du lapin angora manifeste des variations saisonnières se traduisant par la production d'un pelage haut et compact en automne et en hiver, et d'un pelage plus court et plus aéré en été. Le pelage qui pousse au printemps est de nature intermédiaire (Rougeot \& Thebault, 1983). Ces variations portent aussi bien sur les dimensions des poils, quelle que soit leur catégorie, jarres, barbes ou duvets, que sur le nombre de duvets. Les diamètres diminuent légèrement en été par rapport à l'automne - hiver : 3 à 6 p. 100 en passant de la catégorie la plus grossière à la plus fine. L'écart est plus prononcé pour les longueurs, notamment pour les duvets où elles diminuent de 16,8 p. 100 en été par rapport à l'automne-hiver, contre 15,2 p. 100 pour les barbes et 9,9 p. 100 pour les jarres. Mais la différence la plus marquée porte sur le nombre de duvets : le rapport du nombre de duvets à celui des jarres, qui demeure constant, diminue de 28,2 p. 100 en été. On sait (Rougeot \& Thebault, 1983; Rougeot, Thebault \& Allain, 1984) que cette diminution de la population de duvets est la conséquence de la disparition, à la mue de printemps, d'un certain nombre de follicules pileux secondaires dérivés qui se multiplient par bourgeonnement sur le follicule pileux 
principal, du follicule pileux composé. Ainsi, en ce cas, contrairement à ce qui se passe dans le processus classique du cycle folliculaire, il n'y a pas de formation de poil de remplacement avant la chute de l'ancien. A la mue d'automne le nombre de follicules pileux secondaires dérivés se rétablit à la suite de la formation de nouveaux bourgeons pileux. Cette formation adaptative de la composition et de la structure du pelage, observée chez d'autres espèces, n'est pas perturbée par la récolte du poil par épilation qui se répète tous les trois mois, épilation qui possède une action inductrice sur la reprise de la phase d'activité dans le cycle folliculaire et institue une repousse en synchronisme du pelage (CHASE, 1954).

Il résulte de ces diverses modifications saisonnières dans la composition et la structure de la toison du lapin angora que les récoltes d'été fournissent en poids, quelques 25 p. 100 de moins que celles d'automne et d'hiver, celles du printemps étant intermédiaires.

Or il est bien connu que les modifications saisonnières du pelage sont réglées par le photopériodisme (BISSONNETTE, 1935) qui induit des équilibres hormonaux contrôlant les mues et la croissance du pelage. Si on discute encore sur le mécanisme de ce contrôle hormonal (Johnson, 1977 ; Rougeot, Allain \& Martinet, 1984) un fait est désormais acquis, comme cela a été démontré chez l'hermine (Rust \& MEYER, 1969), le hamster Djungarian (Hoffmann, 1973), le vison (Allain \& Rougeot, 1980) et le lapin (BOYD, 1985) : l'administration de mélatonine pratiquée par implants, induit la formation du pelage d'hiver. En est-il de même chez le lapin angora dont la repousse en synchronisme du pelage est induite tous les trois mois par l'épilation?

\section{Matériel et méthodes}

\section{A. Protocole expérimental}

Seize lapines angora, de souche française, âgées de 10 mois, donc adultes, ont été réparties en 2 lots de 8 femelles selon la technique des blocs randomisés en prenant pour critère la quantité de poils produite à la $3^{\mathrm{e}}$ récolte. Les implants de mélatonine ont été introduits sous la peau du flanc gauche des 8 lapines du lot expérimental 7 jours avant la date présumée de la récolte de printemps, la $4^{\mathrm{e}}$, qui eut lieu entre le 15 mai et le 10 juin. La récolte d'été fut effectuée 98 jours après celle de printemps et les implants retirés le jour de la récolte d'été entre le 21 août et le 17 septembre. Les implants de mélatonine, pesés à sec avant la pose étaient constitués de tube silastic de $5 \mathrm{~cm}$ de long ( $\mathrm{n}^{\circ} 602-235 ; 1,47 \mathrm{~mm}$ de diamètre intérieur et $1,96 \mathrm{~mm}$ de diamètre extérieur ; Dow Corning Corporation, Midland Michigan, U.S.A.) et contenaient entre 38,2 et $46,1 \mathrm{mg}$ de mélatonine (Fluka, Bucks, Suisse). La quantité de mélatonine diffusée a été calculée à partir du poids sec des implants retirés.

\section{B. Animaux}

Les animaux ont été maintenus, pendant toute la durée de l'expérience, dans les conditions habituelles de l'élevage, c'est-à-dire élevés sur litière de paille dans des cases individuelles en ciment et dans un local clos où l'éclairage artificiel reproduisait exactement les variations annuelles de la durée d'éclairement correspondant à notre latitude $\left(45^{\circ}\right.$ Nord $)$. 


\section{Alimentation}

L'aliment employé durant l'essai est un aliment granulé standard pour lapin angora. Il contient 17 p. 100 de protéines brutes et 16 p. 100 de cellulose. Il a été distribué, selon le plan de rationnement habituel de l'élevage, à savoir, une distribution journalière pendant 6 jours suivi d'une journée de jeûne et correspondant à une quantité hebdomadaire de $1300 \mathrm{~g}$ durant le mois suivant la récolte de poils, de $1150 \mathrm{~g}$ au cours du $2^{\mathrm{e}}$ mois et de $1000 \mathrm{~g}$ pendant le $3^{\mathrm{e}}$ mois. Cette technique d'alimentation permet d'éviter les enterotoxémies et la formation de trichobezoards (Rougeot \& TheBAult, 1977).

L'abreuvement est assuré en permanence par des abreuvoirs automatiques.

\section{Mesure des caractéristiques de la toison récoltée}

\section{Poids des toisons}

La récolte d'été fut pratiquée 14 semaines après la récolte de printemps, par épilation après traitement au cyclophosphamide (Rougeot \& THEBAULT, 1970). La toison de chaque animal a été pesée au gramme près.

\section{Longueur et diamètre des poils}

Les mesures ont été effectuées sur une mèche prélevée sur le dos de chaque animal immédiatement avant la récolte. Les 3 catégories de poils : jarres, barbes et duvets ont été séparées grâce à l'appareil à pinces déjà décrit (THEBAulT, 1977). Les mesures ont été ensuite effectuées selon les techniques précédemment décrites (THEBault, 1977 ; Rougeot \& Thebault, 1983). Pour les longueurs, les jarres ont été mesurés individuellement en les étendant le long d'une règle graduée à raison de 90 par mèche, tandis que pour les barbes et les duvets, on a simplement mesuré leur hauteur dans la mèche étirée. Les diamètres ont été mesurés sur des fragments de poils de $0,2 \mathrm{~mm}$ de long par projection microscopique (VISOPAN, Reichert, Vienne) selon la norme I.W.T.O. 8-61 (F) (1966) de la Fédération Lainière Internationale : 35 par catégorie de poils de chaque mèche.

\section{Compacité de la toison}

La densité d'implantation des poils a été déterminée à partir du comptage des follicules pileux sur des coupes histologiques de peau effectuées parallèlement à l'épiderme (Rougeot \& Thebault, 1983). L'observation devant être faite au stade anagène, les prélèvements de peau ont été faits 5 semaines après l'épilage de printemps sur le dos de chaque animal avec un emporte pièce de $33 \mathrm{~mm}$. Nous avons compté séparément, sur la projection microscopique des coupes histologiques, d'une part les follicules primaires centraux (PC) producteurs de jarres et éléments permanents aisément reconnaissables et d'autre part les autres follicules pileux primaires latéraux (PL, barbes) et secondaires ( $S$, duvets). Le rapport $\frac{\mathrm{PL}+\mathrm{S}}{\mathrm{PC}}$ permet d'exprimer avec précision la variation de la population des follicules pileux secondaires dérivês puisque la surface de peau prélevée approximative en raison de son élasticité, n'intervient pas. 


\section{Méthodologie statistique}

L'exploitation statistique de tous les paramètres de la toison a été effectuée par analyse de variance.

\section{Résultats}

A. Poids des toisons (fig. 1)

La quantité de poils récoltée, en été, sur les animaux du lot implanté $(275,6 \mathrm{~g} \pm 16,6)$ est significativement $(\mathrm{p}<0,01)$ supérieure $(+31,2 \mathrm{p} .100)$ à celle récoltée sur les animaux témoins $(210,1 \mathrm{~g} \pm 12,8)$.

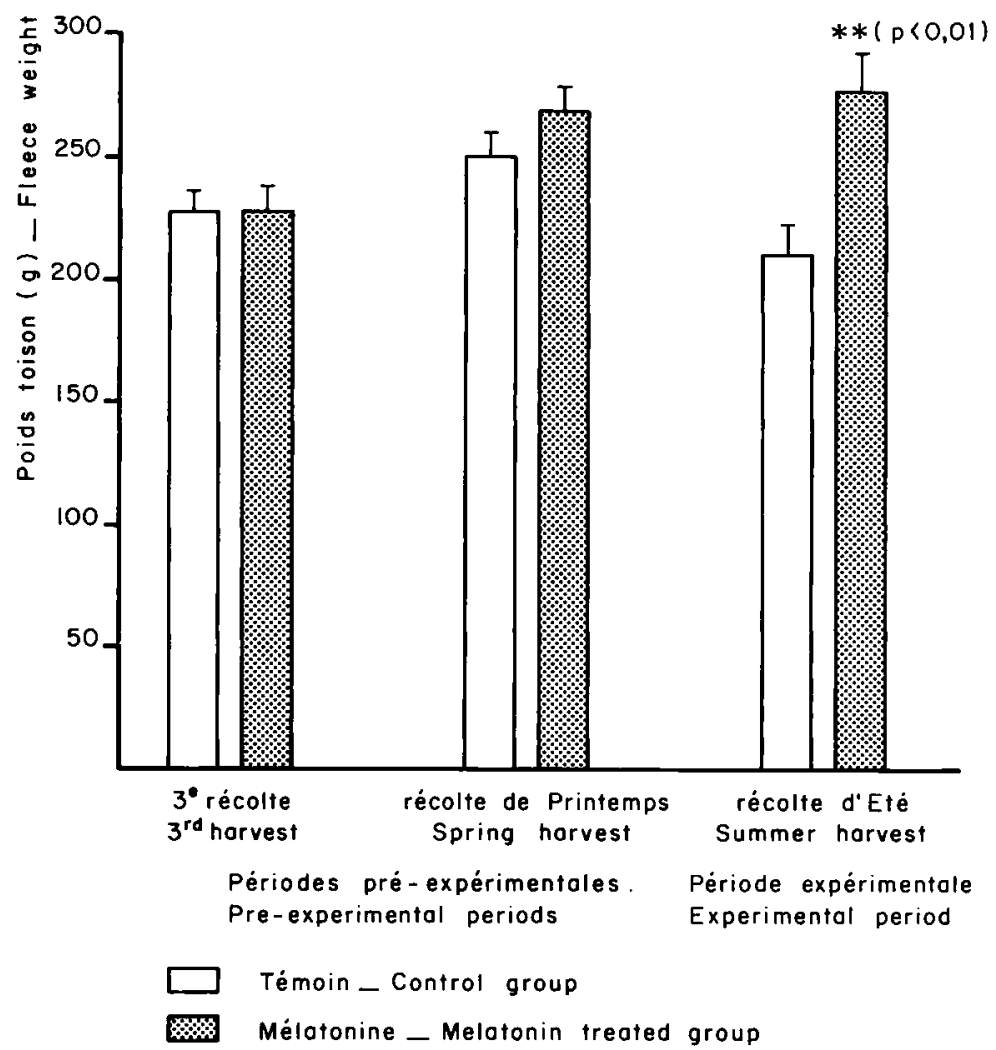

Fig. 1

Productions moyennes de poil angora.

Mean angora wool production. 
Les animaux témoins ont une production d'été inférieure de $16 \mathrm{p}$. 100 à leur propre production de printemps, ce qui correspond aux résultats habituellement observés.

\section{B. Longueur des poils (fig. 2)}

Les poils de couverture, jarres et barbes, du lot implanté sont légèrement plus longs que ceux du lot témoin, respectivement : $10,56 \mathrm{~cm} \pm 0,18$ et $7,85 \mathrm{~cm} \pm 0,15$ contre $10,28 \mathrm{~cm} \pm 0,20$ et $7,48 \mathrm{~cm} \pm 0,21$, mais ces différences ne sont pas significatives.

Par contre, le sous-poil, constitué par les duvets est significativement plus long chez les animaux implantés, $(p<0,01): 6,90 \mathrm{~cm} \pm 0,09$ contre $6,37 \mathrm{~cm} \pm 0,17$.

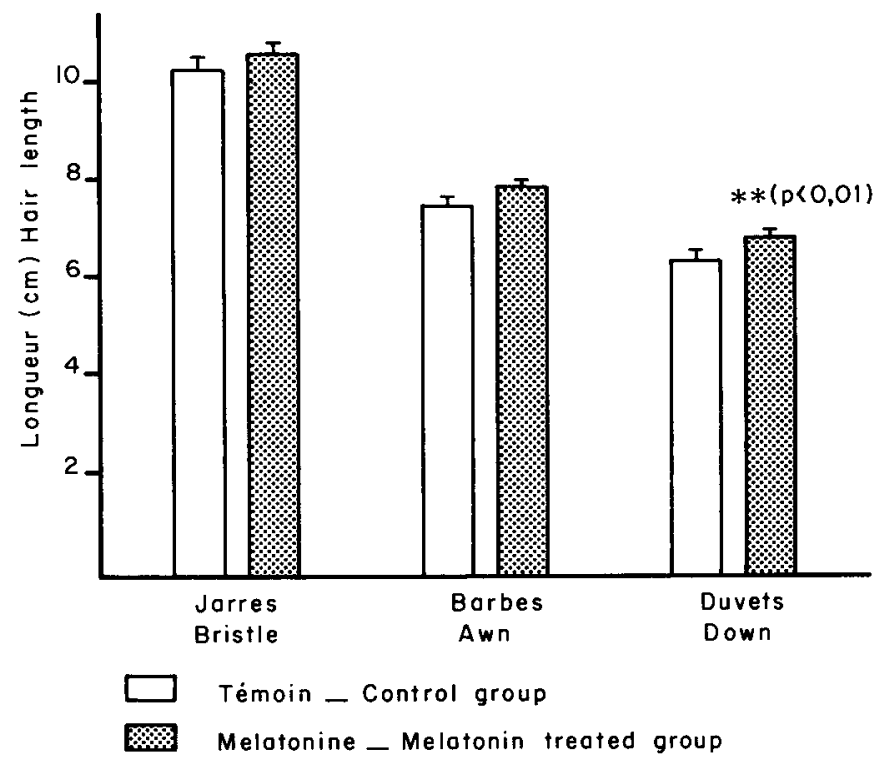

Fig. 2

Longueurs moyennes des 3 catégories de poils.

Mean length of three types of hair.

\section{Diamètre des poils (fig. 3)}

Les diamètres des jarres et des duvets, chez les lapins implantés sont légèrement supérieurs : respectivement $58,23 \mu \mathrm{m} \pm 1,16$ et $13,83 \mu \mathrm{m} \pm 0,17$ contre $55,05 \mu \mathrm{m} \pm 1,19$ et $13,31 \mu \mathrm{m} \pm 0,36$, mais ces différences ne sont pas significatives, alors que le diamètre des barbes est équivalent dans les 2 groupes de lapins $(27,93 \mu \mathrm{m} \pm 0,71$ contre $28,06 \mu \mathrm{m} \pm 0,62$ ). 


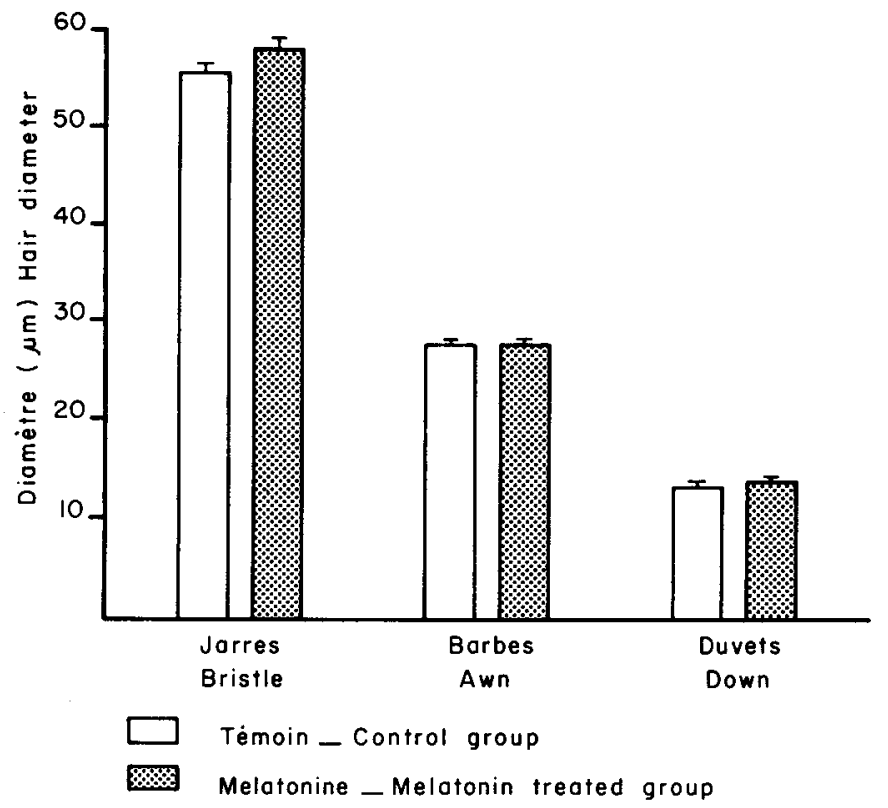

Fig. 3

Diamètre moyen des 3 catégories de poils.

Mean diameter of three types of hair.

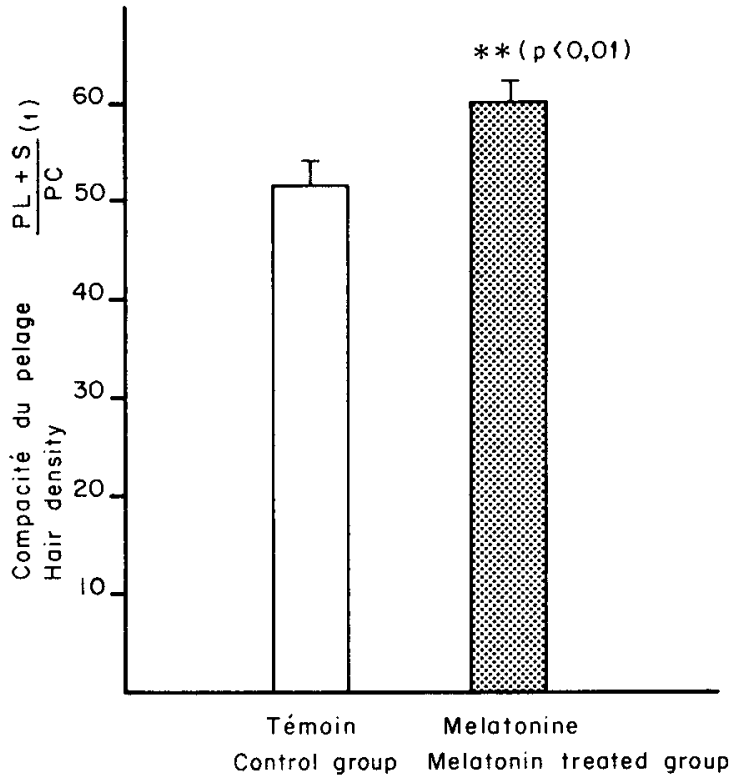

FiG. 4

Compacité du pelage.

Hair density.

(1) PC: Nombre de follicules pileux primaires centraux / Number of central primary follicles. PL : Nombre de follicules pileux primaires latéraux / Number of lateral primary follicles. $\mathrm{S}$ : Nombre de follicules pileux secondaires / Number of secondary follicles. 


\section{Compacité du pelage (fig. 4)}

La densité d'implantation des follicules pileux dans la peau, c'est-à-dire le rapport $\frac{\mathrm{PL}+\mathrm{S}}{\mathrm{PC}}$ est significativement $(\mathrm{p}<0,01)$ plus élevé chez les animaux traités à la mélatonine : $60,3 \pm 2,1$ contre $51,5 \pm 2,6$ chez les animaux témoins, soit une augmentation de 17,1 p. 100 .

\section{E. Diffusion de la mélatonine}

La diffusion de la mélatonine a été contrôlée chez 6 lapins sur 8,2 implants ayant été détériorés lors de leur récupération. La quantité de mélatonine diffusée varie suivant les lapins dans de larges proportions. La perte de poids maximale observée dans les implants retirés s'élève à $4,1 \mathrm{mg}$ tandis que la perte de poids minimale n'est que de $1,3 \mathrm{mg}$, soit un rapport de 1 à 3 . Le poids moyen de mélatonine diffusée est de $2,2 \pm 0,4 \mathrm{mg}$. On constate en outre une corrélation très hautement significative $(r=0,99, p<0,001)$ entre la quantité de mélatonine diffusée et le poids de poils produit.

\section{Discussion}

La pose d'implants de mélatonine chez le lapin angora a permis d'augmenter de façon significative le poids de poils récolté en été et d'effacer la baisse estivale faisant suite à l'épilage de printemps. La différence de 23,8 p. 100 obtenue en moins dans le lot témoin correspond aux chutes de production que l'on constate habituellement à la récolte d'été par rapport à celles d'automne et d'hiver et qui avaient été observées au cours d'une expérience précédente : 25,2 et 27,4 p. 100 respectivement (Rougeot \& Thebault, 1983). D'ailleurs, à la récolte d'automne suivante, 7 lapins expérimentaux, l'un des 8 étant mort entre temps, ont produit en moyenne $277 \mathrm{~g}$ de poils, ce qui est presque identique aux $270,7 \mathrm{~g}$ fournis avec la mélatonine en été par les mêmes lapins.

On peut donc affirmer que la pose d'implants de mélatonine au moment de l'épilage de printemps permet d'obtenir en été une récolte de poils équivalente en quantité à celles d'automne et d'hiver.

En ce qui concerne les dimensions des poils on ne retrouve pas tout à fait les différences obtenues entre été et automne-hiver lors de l'expérience précédente : pas de différence significative entre les diamètres, ni entre les longueurs des jarres et des barbes. Seule la longueur des duvets augmente significativement avec la mélatonine. 
On peut donc avancer deux explications à cette différence de résultats :

1) Le nombre d'animaux observés étant plus réduit, il est plus difficile de mettre en évidence une différence de faible valeur.

2) Les animaux présentent des toisons de caractère légèrement différent, ce qui se constate d'une part aux poids des récoltes de type hivernal, $270 \mathrm{~g}$ dans le cas présent, $230 \mathrm{~g}$ dans l'expérience précédente et d'autre part à la compacité des toisons (rapport $\frac{P L+S}{P C}$ ) de type hivernal, respectivement 60 et 50 . De toute façon ces données ne permettent pas de prétendre que la mélatonine ait une action particulière sur la morphologie des poils.

Cette expérience, et ce n'était d'ailleurs pas son objectif, n'apporte aucun élément nouveau sur le mécanisme d'action de la mélatonine dans l'induction de la mue d'hiver. Il n'en reste pas moins que la proportionnalité directe entre les quantités de mélatonine diffusée et le supplément de poids de poils fourni semble indiquer d'une part que l'on n'a pas obtenu le poids maximal potentiel qu'on serait susceptible d'obtenir avec des doses diffusées supérieures (l'asymptote de production n'a pas été atteinte) et d'autre part que l'action de la mélatonine sur le follicule pileux se fait avec peu d'intermédiaires, sinon directement, comme le soumettent Logan \& WeATHERHEAD (1980).

Quoiqu'il en soit, le traitement des lapins angora par la mélatonine à partir de l'épilage de printemps se révèle intéressant dans la pratique de son élevage. Le coût de cette technique simple peut se justifier par le gain réalisé sur le supplément de production de cette matière textile onéreuse.

Reçu en avril 1986.

Accepté en septembre 1986.

\author{
Summary \\ Use of melatonin implants to prevent reduction of summer wool production \\ in Angora rabbits
}

\begin{abstract}
Adult female Angora rabbits were treated with melatonin implants in May, one week before spring wool harvest and throughout the summer pelage growth ( 98 days); they were kept in a closed building with artificial lighting regulated according to the natural photoperiod by a photoelectric system. The Angora wool production increased by $31.2 \mathrm{p} .100$ in melatonin-treated animals (fig. 1), the hair follicle population by 17.1 p. 100 and down length by 8.3 p. 100 (fig. 2), but the other hair components were not modified (fig. 2 and 3 ).

Use of melatonin implants during summer pelage growth prevented the fall in summer wool production and resulted in a fleece weight similar to that of the autumn and winter.
\end{abstract}

Key words : Angora rabbit, hair production, season, melatonin, coat structure. 


\section{Références bibliographiques}

Allain D., Rougeot J., 1980. Induction of autumn moult in mink (Mustela vison Peale and Beauvois) with melatonin. Reprod. Nutr. Dev., 20, 197-201.

Bissonnette T.H., 1935. Relations of hair cycles in ferrets to changes in the anterior hypophysis and light cycles. Anat. Rec., 63, 159-168.

Boyd I.L., 1985. Effects of photoperiod and melatonin on testis development and regression in wild European rabbits (Oryctolagus cuniculus). Biol. Reprod., 33, 21-29.

Chase H.B., 1954. Growth of the hair. Physiol. Rev., 34, 113-126.

Hoffmann K., 1973. The influence of photoperiod and melatonin on testis size, body weight and pelage color in the Djungarian hamster (Phodopus sungorus). J. Comp. Physiol., 95, 267-282.

Johnson E., 1977. Environmental effects on the hair follicle. In Jarret A., The physiology and pathology of the skin, 4: The Hair follicle, 1389-1415. Acad. Press, London.

Logan A., Weatherhead B., 1980. Post tyrosinase inhibition of melanogenesis by melatonin in hair follicles in vitro. $J$. Invest. Dermatol, 74, 47-50.

Rougeot J., Thebault R.G., 1970. Utilisation du cyclophosphamide comme substance dépilatoire pour la récolte des poils du lapin angora. Ann. Zootech., 19, 229-234.

Rougeot J., Thebault R.G., 1977. Formation de trichobezoards chez le lapin angora nourri ad libitum avec un aliment aggloméré. Rec. Med. Vet., 153, 655-659.

Rougeot J., Thebault R.G., 1983. Variations saisonnières de la composition et la structure du pelage : exemple de la toison du lapin angora. Ann. Zootech., 32, 287-314.

Rougeot J., Allain D., Martinet L., 1984. Photoperiodic and hormonal control of seasonal coat changes in mammals with special reference to sheep and mink. Acta Zool. Fennica, 171, 1318.

Rougeot J., Thebault R.G., Allain D., 1984. Role of the compound hair follicle in adaptative pelage changes. Acta Zool. Fennica, 171, 19-21.

Rust C.C., MeYer K.K., 1969. Hair color, moult and testis size in male short tailed weasels treated with melatonin. Science, 165, 921-922.

Thebault R.G., 1977. Le lapin angora. Développement post-natal de sa toison; variation saisonnière de la production de poils. Mémoire D.P.E., Cons. Nat. Arts et Métiers, Paris, 105 pp. 